\title{
Essais
}

Revue interdisciplinaire d'Humanités

Hors-série 7 | 2022

Récits d'outre-thèse

\section{Entre estrangement et sérendipité : première année d'une vie de docteure}

Between estrangement and serendipity: The first year of a doctoral life

\section{Rime Fetnan}

\section{(2) OpenEdition}

Journals

Édition électronique

URL : https://journals.openedition.org/essais/10832

DOI : 10.4000 /essais. 10832

ISSN : 2276-0970

Éditeur

École doctorale Montaigne Humanités

\section{Édition imprimée}

Date de publication : 15 avril 2022

ISBN : 979-10-970024-00-0

ISSN : $2417-4211$

Référence électronique

Rime Fetnan, «Entre estrangement et sérendipité : première année d'une vie de docteure », Essais [En ligne], Hors-série 7 | 2022, mis en ligne le 01 février 2022, consulté le 18 janvier 2023. URL : http:// journals.openedition.org/essais/10832 ; DOI : https://doi.org/10.4000/essais.10832

Ce document a été généré automatiquement le 18 janvier 2023.

Tous droits réservés 


\section{Entre estrangement et sérendipité : première année d'une vie de docteure}

Between estrangement and serendipity: The first year of a doctoral life Rime Fetnan

\section{Préambule à la soupe}

1 Quand je reçois l'appel à contribution du numéro d'Essais consacré aux « récits de docteurs ", je m'interroge : comment pourrais-je synthétiser - et surtout concrétiser à travers le récit - la multitude d'événements et de ressentis que j'ai traversés depuis le jour où je suis devenue docteure? Mon expérience me parait à la fois anecdotique et trop complexe pour être pertinemment narrée. En plus de cela, je trouve l'exercice plutôt narcissique pour une revue universitaire. Et pour finir, j'ai l'impression que la perspective avec laquelle j'aborde cette expérience du post-doctorat est tellement déprimante et sans recul qu'il n'y aurait aucune grande leçon à tirer de tout cela. Pourtant, je décide de me prêter à l'exercice. Par ennui peut-être, pendant que ma soupe mijote, ou peut-être parce que je suis finalement assez narcissique.

2 C'est sans doute ce même narcissisme qui m'a poussée à m'inscrire en doctorat, et à assumer les désirs d'élévation sociale et intellectuelle transmis par ma famille d'immigrés. Ce syndrome de la "bonne élève", conjugué à mon incapacité à me projeter dans le monde du travail au sortir du master, associé à un contrat doctoral miraculeusement obtenu après un refus d'inscription pour note insuffisante, ont été les ingrédients qui m'ont conduite à réaliser cette extraordinaire tambouille que l'on appelle la thèse.

3 Je crois que je ne me suis jamais vraiment considérée comme chercheuse, encore moins comme une intellectuelle. Même s'il y a eu une époque glorieuse - entre mes 7 et mes 14 ans - durant laquelle je lisais autant de livres que mon temps libre me le permettait, 
ma vie d'adulte a plutôt été tournée vers la recherche d'expériences sociales passionnantes. Une grande partie de cette vie d'adulte a d'ailleurs été logiquement consacrée à essayer d'apaiser - par le biais de nombreuses séances de psychanalyse - ce sentiment d'imposture qui découlait de ces plaisirs superficiels, ainsi qu'à dénouer une multitude de névroses qui ne sont pas le sujet de ce récit. Malgré cela, et grâce à un contexte marqué par un environnement de travail idéal, j'ai fait en sorte que mon expérience du doctorat soit la plus enrichissante possible, et de me forger une « solide expérience de la recherche ", pour paraphraser mon curriculum vitae.

Avant de m'atteler à la narration des faits s'étant déroulés après l'obtention de mon doctorat - j'ai conscience d'être restée pour l'instant un peu hors sujet - je tiens à préciser que pendant toute la durée de ma thèse j'ai, je l'avoue, jugé assez durement en mon for intérieur les autres docteurs qui vivaient un désarroi psychologique au lendemain de la soutenance. Non pas que je n'étais pas consciente de la grande précarité dans laquelle nous sommes quasiment tous et toutes plongés lorsque les murs protecteurs de l'université sont tombés, mais plutôt que j'étais dans l'incapacité de comprendre ce qu'il y avait de difficile à retourner à la « vraie vie » après des années de frustrations et de sacrifices. Naïvement, j'étais persuadée que je n'aurais aucun mal à m'adapter à mon nouveau quotidien, auréolée par le succès de ma soutenance, pensant que la reconnaissance de ma famille et de mes pairs suffirait à me combler jusqu'à la fin de mes jours.

\section{La « dame du CDI »}

5 Le premier choc est survenu lors de ma rentrée en tant que professeure-documentaliste remplaçante dans un lycée, la rentrée qui a suivi ma soutenance. J'étais en train de me laver les mains dans les toilettes des enseignants, lorsqu'une professeure, visiblement à la recherche d'une personne sur laquelle exercer son autorité limitée, m'ordonne de quitter les lieux. Au mois de juin je me tenais au milieu d'une assemblée d'amis, collègues et professeurs réunis solennellement pour ma soutenance, un verre de champagne à la main, discutant de mon sujet sur la fabrique des imaginaires de l'altérité dans les biennales internationales d'art contemporain depuis 1989, et trois mois plus tard, je suis confrontée très violemment à cette altérité qui me prend pour une lycéenne qui utilise les mauvaises toilettes.

6 J'ai réalisé très rapidement que la fonction de « dame du CDI» (pour citer les lycéens), n'est pas idéale pour entamer une vie professionnelle - aussi alimentaire qu'elle soit. En effet, elle est à la fois trop semblable aux fonctions d'enseignante à l'université, et en même temps trop éloignée du sentiment d'accomplissement que j'avais lorsque j'exerçais ces fonctions. J'étais comme projetée dans la version médiocre et insatisfaisante de ma vie lorsque j'étais doctorante: je faisais des ateliers de méthodologie, mais il fallait d'abord convaincre l'enseignant titulaire moins diplômé que moi que j'étais tout à fait apte à aider les élèves; mes collègues me demandaient si j'avais soutenu mon mémoire et si je comptais présenter le concours de professeure documentaliste dans l'année; et certains élèves qui pensaient que la terre était plate considéraient que mes fonctions ne me conféraient pas le sens critique nécessaire pour leur apprendre quoi que ce soit.

7 Le fait le plus étrange a été mon incapacité à considérer cette expérience comme transitoire : alors que la situation aurait pu me conforter dans mon souhait d'embrasser 
une carrière d'enseignante-chercheuse, et de m'élever au-dessus de toute cette trivialité qui me frappait dans sa réalité la plus crue, je me suis vue, dans 10 ans, ressembler à ma collègue Sylvie*, dont le quotidien est rythmé par la commande de livres que les élèves ne liront jamais, le café de $10 \mathrm{~h} 30$ avec Claude* la gestionnaire de l'établissement, et la surveillance des lycéens qui auraient l'audace d'introduire des kitkats dans l'enceinte de ce lieu sacré qu'est le CDI.

Je pense que si j'ai été incapable de considérer que « dame du CDI » n'était qu'une étape - certes un peu douloureuse, mais facilement surmontable - de ma longue carrière d'intellectuelle, c'est justement parce que je ne me considérais pas comme une intellectuelle: non pas que je n'avais pas les qualités requises, mais plutôt parce que j'étais soudain frappée d'une énorme flemme de me battre. En deux mois, j'avais passé plus de temps à feuilleter les magazines Okapi et 01net à la recherche de l'abonnement fibre avec le meilleur rapport qualité prix, plutôt qu'à écrire des articles.

\section{"Mais alors, qu'est-ce que vous faites là si vous êtes docteure?»}

9 Le problème, c'est surtout que je me suis considérée justement et à tort comme une "docteure ", et uniquement comme cela. Le fait qu'un diplôme devienne un statut si totalisant (sans que la reconnaissance sociale, c'est-à-dire une profession, découle de ce statut, contrairement aux médecins par exemple, qui sont docteurs de diplôme et de métier), est en réalité très enfermant. Une prison dorée, car cette appellation à elleseule est censée procurer un sentiment d'accomplissement. Pire encore, elle nous donne l'impression que nous ne sommes pas seuls, comme si tous les docteurs faisaient partie d'une communauté, liés par une expérience quasi mystique qui nous distinguerait du reste de la société, gonflés par la conviction d'appartenir à l'élite intellectuelle. Puisque le diplôme de « docteur » était semblable à un statut honorifique comme celui de "professeur émérite", j'avais l'impression de faillir à mes responsabilités, m'abaissant à me contenter de mon poste dans le secondaire, alors que la seule évocation de ma thèse dans mes cercles amicaux, familiaux et professionnels, suffisait à me faire passer sans que je le veuille pour la personne la plus intelligente de la pièce.

10 Je n'étais visiblement pas la seule à trouver ce décalage entre mon niveau d'étude et ma situation professionnelle dérangeante, voire improbable : quelques semaines après mon inscription à Pôle emploi, j'ai été convoquée pour une formation obligatoire pour tous les nouveaux inscrits et qui visait à m'apprendre à utiliser un ordinateur. Dans le descriptif, je prends connaissance avec plus de détails du contenu de cette formation censée m'occuper une matinée entière : démarrer un ordinateur; déplacer la souris ; rechercher un réseau wifi ; etc. Considérant que j'avais mieux à faire, après six années passées à rédiger un manuscrit de 450 pages (annexes non comprises), j'ai demandé l'autorisation à ma conseillère d'être dispensée de cette formation, ce à quoi elle a opposé un refus catégorique. Je précise que le débat houleux qui s'en est suivi s'est tenu en plein milieu du hall d'accueil de Pôle emploi, et qu'il n'a pas fallu longtemps pour que mes concitoyens chômeurs nous fixent, ma conseillère et moi, en train d'argumenter sur la non-nécessité d'une telle formation. Avec du recul, je considère ce débat comme ayant été plus difficile à mener que les nombreuses expériences de colloques internationaux que j'ai pu avoir. Au bout d'un moment, agacée par le 
caractère obtus de cette conversation qui semblait sans fin, je fais appel à mon statut honorifique jusque-là resté sous silence: je lance à ma conseillère que je suis "docteure », espérant lire dans ses yeux un quelconque signe ou au moins début de considération et de compréhension. Au lieu de cela, elle me répond, visiblement très interloquée : «mais alors, qu'est-ce que vous faites là, si vous êtes docteure?»

\section{«T'as qu'à faire une deuxième thèse "}

Mes proches, à qui j'avais fait subir les aléas émotionnels d'une thésarde en mal de confiance en elle, furent assez découragés en constatant que je vivais l'après-thèse comme une crise d'adolescence tardive, oscillant entre profonde déprime et euphorie pour des activités improbables comme l'astrologie ou la plongée sous-marine en plein hiver. Certains à qui je décrivais le sentiment de vide qui m'habitait me conseillaient même de "faire une deuxième thèse ", comme si cela pouvait solutionner mon manque de perspectives.

Pourtant ce n'était pas la précarité qui me gênait le plus: je n'étais pas davantage convaincue de vouloir obtenir à tout prix un poste de maître de conférences, ce qui aurait impliqué de déménager dans une ville peu attrayante, de gagner à peine plus qu'un smic en début de carrière et de devoir être confrontée à des luttes de pouvoir incessantes dans un laboratoire de recherche qui cherche par tous les moyens à être le plus compétitif. De plus, je bénéficiais d'une situation financière convenable - bien que précaire - qui suffisait pour ma situation de célibataire sans enfants à charge. Et comme on me le faisait remarquer à juste titre, mon découragement n'était pas si exceptionnel dans la mesure où beaucoup de «jeunes » diplômés étaient confrontés à la désillusion face au monde du travail qui est en somme très différent du havre que constitue l'université.

13 Mon malaise tenait surtout au fait que j'avais l'impression d'être un sportif de haut niveau qui, au summum de ses capacités physiques, doit brutalement s'arrêter à cause d'une blessure. L'aventure intellectuelle que j'avais vécue pendant mes années de doctorat avait forgé une détermination qui m'avait permis de mener mes recherches et l'écriture de ma thèse pendant six ans et qui avait laissé place à une grande lassitude, une incapacité à mobiliser mes forces dans la poursuite du parcours. J'avais été formée à répondre à des attentes multiples, à conjuguer la recherche, l'écriture, l'enseignement, et la confrontation régulière de mon travail à mes pairs et le monde d'après me paraissait rompre avec ces exigences. J'avais conduit ma réflexion à des niveaux élevés de compréhension du monde, j'avais inondé mon cerveau de quantité d'informations complexes à comprendre et à retenir, j'avais élaboré un discours complexe, logique et dense. Le fait d'être extraite du milieu universitaire signifiait pour moi intégrer un quotidien beaucoup plus trivial, que je considérais comme lent et médiocre.

\section{Cultiver son propre jardin}

14 Alors que je sombrais chaque jour un peu plus dans un état dépressif qui n'avait rien de romantique, une amie m'a proposé de passer quelques jours dans la maison de ses parents qui habitent dans l'arrière-pays niçois. Son père, retraité, consacre presque tout son temps à l'entretien de son immense potager, dont il me fait faire le tour, 
m'expliquant quelques-unes des ficelles de la permaculture, tout en longeant les allées chargées de fruits et légumes. En l'écoutant je réalise à quel point il est difficile de recréer le chaos subtil de la nature, la main de l'homme venant inévitablement déranger l'équilibre fragile du cycle de la terre. Je trouve qu'il y a quelque chose de très humble et en même temps de très courageux dans cette idée que chaque geste s'inscrit dans un processus naturel, processus qui le contraint à n'être ni trop en avance, ni trop en retard. Je comprends que dans la permaculture, les idées de productivité et de rentabilité sont réinterrogées, au profit de l'observation et de l'accompagnement des processus naturels. Cela demande évidemment beaucoup d'énergie et de temps car il y a des milliers de choses différentes à faire : tailler tel élément, protéger tel autre des attaques de frelons et d'oiseaux, épaissir le sol pour conserver l'humidité, arroser (ni trop ni pas assez). J'admire cette capacité à maîtriser le jeu de la vie qui consiste à trouver l'équilibre subtil entre l'appréciation du moment présent et l'anticipation de ce qui doit être fait pour que le cycle se perpétue. En tant que citadine et bonne sceptique, j'ai toujours trouvé les métaphores de développement personnel comme «cultiver son propre jardin » complètement naïves et pourtant, me voilà à trouver du réconfort dans la cueillette de tomates. Je repense aux paroles de l'un de mes mentors de doctorat, Sandro Landi, qui nous encourageait à nous ouvrir à la sérendipité : à nous laisser surprendre par des hasards, à nous ouvrir à des domaines a priori éloignés de nos objets de recherche pour nourrir notre démarche intellectuelle.

C'est alors que je me rends compte que je suis tombée dans une forme de déséquilibre, que je suis à la fois dans une anticipation trop lointaine, assommée par l'angoisse de devoir trouver une porte de sortie à la précarité, et dans l'incapacité d'apprécier (dans le sens d'estimer, d'évaluer) les choses qui se présentent à moi sur le moment. Et que du coup, rien ne pousse.

16 Moi qui ai passé des années à étudier le concept d'altérité, je suis désormais confrontée à la difficulté de reconnaître une part de ma propre étrangeté : qui suis-je, quelle est ma place, et quel est mon but une fois le travail accompli ? Plus encore, je réalise que mon entreprise de déconstruction des identités figées et essentialisées dans le champ de l'art contemporain trouve écho à celle que je mène pour mon propre compte. Il est désormais nécessaire que je me pense hors du cadre que je m'étais construit, que je m'interroge sur les multiples facettes de ma personnalité, en somme, que je fasse preuve d'estrangement.

Certes, il y a quelque chose de vertigineux, voire de très déstabilisant dans le fait de constater que l'on est étranger à soi-même à 30 ans. Mais mon statut de docteure, justement parce qu'il est immuable et qu'aucune fonction ou un métier précis n'en découle obligatoirement, me permet de renouveler l'image que j'avais de moi-même, tout en me donnant l'assise nécessaire pour envisager les possibles.

* Pour préserver l'anonymat de mes chères collègues, les prénoms ont été modifiés. 


\section{AUTEUR}

\section{RIME FETNAN}

Docteure en Communication, Arts et Spectacles

Université Toulouse II Jean Jaurès 